\title{
REAL BOREL COHOMOLOGY OF LOCALLY COMPACT GROUPS
}

\author{
BY \\ ARTHUR MASON DUPRE III(')
}

INTRODUCTION

If mathematical problems be considered pure in inverse proportion to their history, those herein considered may justifiably be though of as reasonably pure. One of the prime motivations for the subject of this thesis has been the availability, thanks to Dr. Calvin Moore, of a handwritten manuscript which has been in my possession for two years, which is his doctoral dissertation, just published in [16]. A major portion of the following paper is concerned with laying firmer foundations for the theory of Borel cohomology, in the belief that this will hasten defeat of the enemy. Some of the theorems in the first two sections are modeled on those of Snapper, in [18], who considered these problems for everything discrete, the powerful techniques of homological algebra being available in this case. Unfortunately, there is no machinery of projective or injective resolutions immediately at our disposal for the cohomology theories based on Borel cochains. There is a type of resolution, which leans toward the injective, but its full homological character is not understood as yet. The entire idea of considering the cohomology of general group actions was inspired by the paper of Snapper. These things have been considered in the continuous case by Mostow in [17], where equivariant cohomology groups are defined and studied.

Almost nothing being known about the higher cohomology groups, it was desirable to ferret out those isolated pockets of triviality. This is done in the last section, where it is shown that for $\boldsymbol{G}$ abelian locally compact separable metrizable, $\boldsymbol{R}$ the real line, $\boldsymbol{G}$ operating trivially on $\boldsymbol{R}, \bar{H}^{n}(\boldsymbol{G}, \boldsymbol{R})$, the bounded Borel groups defined in [16], do not depend upon the topology of $G$, nor its torsion. In fact, $R$ seems to ignore everything about $\boldsymbol{G}$ except a certain torsion-free discrete group $D$ associated to $\boldsymbol{G}$.

The further fact that these groups are isomorphic to the group of alternating multilinear functions $\bigwedge^{n}(\boldsymbol{G}, \boldsymbol{R})$ was suggested by the results of Kleppner on $H^{2}(G, T)$, where $G$ is locally compact abelian and $T$ the circle group. He showed that for all $\boldsymbol{G}$ except a certain intractable class of 2-primary groups, whose fate is yet undecided, that $H^{2}(G, T)=L^{2}(G, T) / S^{2}(G, T)$, the continuous bihomomorphisms modulo the symmetric ones. We have not resolved this open question,

Received by the editors December 13, 1965 .

(1) Thesis submitted to the Faculty of the Graduate School of the University of Maryland in partial fulfillment of the requirements for the degree of Doctor of Philosophy. 
but can show that $H_{c}^{2}(G, T)=H^{2}(G, T)$, the group on the left being the second continuous cohomology group, and $\boldsymbol{G}$ being a certain type of solvable group, including all abelian groups.

As for matters of notation, everything is pretty well standard, except that we write $\lim ^{i}, \lim _{i}$ for injective and projective limits.

\section{ChAPTER I. The Cohomology Groups}

$X$ is a separable space complete with respect to some metric furnishing its topology, hereafter referred to as a polonais space. There are also on the scene two groups $\boldsymbol{G}, \boldsymbol{A}$, of which $\boldsymbol{G}$ is polonais, $\boldsymbol{A}$ is abelian and complete metrizable. $\boldsymbol{G}$ operates on $\boldsymbol{X}$ and $\boldsymbol{A}$ by means of continuous maps $\boldsymbol{G} \times \boldsymbol{X} \rightarrow \boldsymbol{X}, \boldsymbol{G} \times \boldsymbol{A} \rightarrow \boldsymbol{A}$, where for fixed $g \in G, x \rightarrow g x$ is a homeomorphism of $X, a \rightarrow g a$ is a topological automorphism of $A$, and by operate we mean that $g_{1}\left(g_{2} x\right)=\left(g_{1} g_{2}\right) x, g_{1}\left(g_{2} a\right)$ $=\left(g_{1} g_{2}\right) a$, for all $\left(g_{1}, g_{2}, x, a\right) \in \boldsymbol{G} \times \boldsymbol{G} \times \boldsymbol{X} \times \boldsymbol{A}$. We call $\boldsymbol{A}$ a $\boldsymbol{G}$-module and $\boldsymbol{X}$ a $\boldsymbol{G}$-space. $(\boldsymbol{G}, \boldsymbol{X})$ denotes $\boldsymbol{G}, \boldsymbol{X}$ together with an operation of $\boldsymbol{G}$ on $\boldsymbol{X}$. A morphism $(f, \alpha):(\boldsymbol{G}, \boldsymbol{X}) \rightarrow(\boldsymbol{H}, \boldsymbol{Y})$ is a pair of maps such that $f: \boldsymbol{G} \rightarrow \boldsymbol{H}$ is a continuous homomorphism and $\alpha: X \rightarrow \boldsymbol{Y}$ is either continuous or Borel, defined below, depending upon the context. Further we require that $\alpha(g x)=f(g) \alpha(x)$ for all $(g, x) \in \boldsymbol{G} \times \boldsymbol{X}$. $\boldsymbol{G}$ acts coordinatewise on $\boldsymbol{X}^{n}$, the action being defined by $g\left(x_{1}, \ldots, x_{n}\right)=\left(g x_{1}, \ldots, g x_{n}\right)$. We shall define three classes of cohomology groups, and under certain conditions, obtain a spectral sequence for each class, similar to that of Moore in [16], for a closed normal subgroup of a polonais locally compact group. Pursuant of this, we continue to make definitions.

A subset of a space is called a Borel set if it lies in the $\sigma$-algebra generated by the open sets. A function $f: \boldsymbol{X} \rightarrow \boldsymbol{Y}$ is called Borel if the inverse image under $f$ of a Borel set is Borel, and bounded if it takes compact sets into precompact sets. A section for $f$ is a function $s: \boldsymbol{Y} \rightarrow \boldsymbol{X}$ such that $f \circ s=1_{Y}$, the identity map on $\boldsymbol{Y}$. For $n>0$, define $\boldsymbol{F}^{n}(\boldsymbol{X} ; \boldsymbol{G}, \boldsymbol{A})=\left\{f: \boldsymbol{X}^{n} \rightarrow \boldsymbol{A}\right\}, \boldsymbol{B}^{n}(\boldsymbol{X} ; \boldsymbol{G}, \boldsymbol{A})=\left\{f \mid f\right.$ is Borel $\left.\cap \boldsymbol{F}^{n}\right\}$, $\bar{B}^{n}(\boldsymbol{X} ; \boldsymbol{G}, \boldsymbol{A})=\left\{f \mid f\right.$ is bounded $\left.\cap \boldsymbol{B}^{n}\right\}, \boldsymbol{C}^{n}(\boldsymbol{X} ; \boldsymbol{G}, \boldsymbol{A})=\left\{f \mid f\right.$ is continuous $\left.\cap \boldsymbol{F}^{n}\right\}$, where $\boldsymbol{X}$ is a $\boldsymbol{G}$-space, $\boldsymbol{A}$ a $\boldsymbol{G}$-module, and the $\boldsymbol{G}$ appearing in the above definitions denotes the fact that each of these groups is a $G$-module with the following operation: $(g f)(x)=g\left(f\left(g^{-1} x\right)\right)$ for all $(g, x)$ in $\boldsymbol{G} \times \boldsymbol{X}$.

On $\boldsymbol{F}^{n}$, we take as a basis for a uniformity the sets $\{(f, g) \mid(f(x), g(x)) \in V$, for $x$ in $K, K$ compact in $X^{n}, V$ open and in the uniformity of $\left.A\right\}$, where the sets $\{(x, y) \mid x-y \in U, U$ an open neighborhood of 0 in $A\}$ are a basis for the uniformity of $\boldsymbol{A}$. This uniformity is compatible with the group structure on $\boldsymbol{F}^{n}$ of pointwise addition of functions. The neighborhoods of 0 for the topology of this uniformity have as a basis the sets of the form $\left\{f \mid f(K) \subset U, K\right.$ compact in $X^{n}, U$ an open neighborhood of 0 in $\boldsymbol{A}\}$. If $\boldsymbol{X}$ is locally compact, then it is $\sigma$-compact and the above groups are all metrizable with the relative topology, and in any case complete, since the Borel functions are closed under sequential pointwise convergence, which suffices, since the range of these functions is metrizable and the convergence is 
uniform on compact sets. It would be interesting, and useful if natural, to find a topology on $\boldsymbol{B}^{n}$ or even $\overline{\boldsymbol{B}}^{n}$ which was at once complete, metrizable, separable, and compatible with the group structure. For proofs of these statements on completeness, see [8, p. $68 \mathrm{ff}$.]. $X^{G}$ denotes the set of $x$ in $X$ such that for all $g$ in $G, g x=x$. The above defined operation of $G$ on $F^{n}, B^{n}, \bar{B}^{n}, C^{n}$, is continuous and so $\left(\boldsymbol{F}^{n}\right)^{G},\left(\boldsymbol{B}^{n}\right)^{G},\left(\bar{B}^{n}\right)^{G},\left(\boldsymbol{C}^{n}\right)^{G}$ are all closed. Define $\partial_{n}: \boldsymbol{F}_{u} \rightarrow \boldsymbol{F}^{n+1}$ as $\partial_{n} f\left(x_{1}, \ldots, x_{n+1}\right)$ $=\sum_{k=1}^{n+1}(-1)^{k+1} f\left(x_{1}, \ldots, \hat{x}_{k}, \ldots, x_{n+1}\right)$, where $\hat{x}_{k}$ means that the variable $x_{k}$ is to be omitted.

LEMMA. $\partial_{n}:\left(F^{n}\right)^{G} \rightarrow\left(F^{n+1}\right)^{G},\left(B^{n}\right)^{G} \rightarrow\left(B^{n+1}\right)^{G},\left(\bar{B}^{n}\right)^{G} \rightarrow\left(\bar{B}^{n+1}\right)^{G},\left(C^{n}\right)^{G} \rightarrow\left(C^{n+1}\right)^{G}$, and is continuous for the topologies involved.

Proof. $\delta_{k}:\left(F^{n}\right)^{G} \rightarrow\left(F^{n+1}\right)^{G}, \delta_{k} f\left(x_{1}, \ldots, x_{n+1}\right)=f\left(x_{1}, \ldots, x_{k}, \ldots, x_{n+1}\right)$ is continuous because it is induced from the continuous map $\left(x_{1}, \ldots, x_{n+1}\right) \rightarrow$ $\left(x_{1}, \ldots, x_{k}, \ldots, x_{n+1}\right)$ of $X^{n+1} \rightarrow X^{n}$ and $\partial_{n}$, as a sum of continuous maps, is also.

COROLlaRY. $Z_{d}^{n}=\operatorname{ker} \partial_{n} \cap\left(F^{n}\right)^{G}, Z^{n}=Z_{d}^{n} \cap\left(B^{n}\right)^{G}, \quad \bar{Z}^{n}=Z_{d}^{n} \cap\left(\bar{B}^{n}\right)^{G}, \quad Z_{c}^{n}=Z_{d}^{n}$ $\cap\left(C^{n}\right)^{G}$ are all closed.

DEFINITION. We define the cohomology groups as usual, as the kernel of $\partial_{n+1}$ modulo the image of $\partial_{n}$, and the various groups thus arising are denoted $H_{d}^{n}(X ; G, A)$, $H^{n}(\boldsymbol{X} ; \boldsymbol{G}, \boldsymbol{A}), H^{n}(\boldsymbol{X} ; \boldsymbol{G}, \boldsymbol{A}), H_{c}^{n}(\boldsymbol{X} ; \boldsymbol{G}, \boldsymbol{A})$.

These groups are not in general Hausdorff with the quotient topology, though in a great many cases it can be verified that they are, and are called respectively the discrete, Borel, bounded Borel, and continuous groups.

We now restrict ourselves to those $(G, X)$ for which $Y=X / G$, the space of orbits, is polonais in the quotient topology. Then the quotient map $\varphi: X \rightarrow Y$, being continuous, open and onto, has a Borel section $s$ which is therefore a Borel isomorphism of $Y$ with $s(Y)$. See [10, p. 397]. $s(Y)$ is not necessarily polonais, although always Borel. $\varphi_{2}: G \times X \rightarrow X$, being continuous, is also when we restrict to $\boldsymbol{G} \times s(\boldsymbol{Y})$. We require that this restriction, which is onto $X$, have a Borel section $t=\left(t_{1}, t_{2}\right)$. This also gives a section $u: X \rightarrow G \times Y$ of $\varphi_{3}: G \times Y \rightarrow X$, where these maps are related in the commutative diagram below.

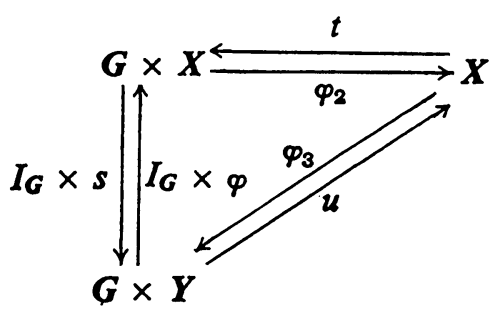

Our next theorem gives a technique for descending from $\boldsymbol{G}$-maps on $\boldsymbol{X}$ to maps on $\boldsymbol{Y}$. 
THEOREM. There is an algebraic isomorphism between $B^{1}(X ; G, A)^{G}$ and the set of Borel functions $f: \boldsymbol{Y} \rightarrow \boldsymbol{A}$ such that for all $y$ in $\boldsymbol{Y}, f(y) \in \boldsymbol{A}_{s(y)}=\boldsymbol{A}^{G}(y)$, where $\boldsymbol{G}_{x}$ is the isotropy subgroup at $x$ in $X$.

Proof. If $f$ is in $B^{1}(X ; G, A)^{G} \equiv B(X, A)^{G}$, define $\alpha f=f \circ s$; then $\alpha f$ is a Borel function from $Y$ to $A$ such that $f(y)$ is in $A_{s(y)}$ for $y$ in $Y$, since $f(x)$ is in $A_{x}$ for $x$ in $X$. Conversely, if $f$ is Borel from $Y$ to $A$ satisfying the above condition, define $(\beta f)(x)=t_{1}(x) f\left(s^{-1}\left(t_{2}(x)\right)\right), f$ is clearly Borel but we must check that $(\beta f)(g x)$ $=g((\beta f)(x))$. We claim that $t_{2}(g x)=t_{2}(x)$. Indeed, let $x$ be in $X$ and write $x=$ $t_{1}(x) t_{2}(x)$; then $g x=\left(g t_{1}(x)\right) t_{2}(x)$ which also is $t_{1}(g x) t_{2}(g x)$. Now because $t_{2}(x)$ and $t_{2}(g x)$ are either in different orbits of $\boldsymbol{X}$ or identical, they must coincide, and we have justified the claim. From the above equations, it follows that $g t_{1}(x)$ and $t_{1}(g x)$ have the same effect on $t_{2}(x)=t_{2}(g x) .(\beta f)(g x)=(\beta f)\left(t_{1}(g x) t_{2}(g x)\right)=$ $t_{1}(g x) f\left(s^{-1}\left(t_{2}(g x)\right)\right)$. Now $f\left(s^{-1}\left(t_{2}(x)\right)\right)$ is in $A_{s\left(s^{-1}\right.}{ }_{\left.\left(t_{2}(x)\right)\right)}=A_{\left.t_{2}(x)\right)}$, so the string of equalities continues with $t_{1}(g x) f\left(s^{-1}\left(t_{2}(x)\right)\right)=g t_{1}(x) f\left(s^{-1}\left(t_{2}(x)\right)\right)=g(\beta f(x))$. It is a straightforward task to verify that $\alpha$ and $\beta$ are inverses of one another.

Following Snapper in [18], we now investigate the effect of a map $\varphi: A_{1} \rightarrow A_{2}$ of $G$-modules on the induced map $\varphi_{*}$ mapping $B\left(X, A_{1}\right)^{G} \rightarrow B\left(X, A_{2}\right)^{G}$. If $\varphi$ is one to one, so is $\varphi_{*}$; if $\varphi$ is an isomorphism, $\varphi_{*}$ is too. The most interesting case is that when $\varphi$ is onto.

Definition. If $\varphi: A_{1} \rightarrow A_{2}$ is a continuous onto $G$-module map, a separable subset in $A_{2}$ is called admissible if there is a separable subset of $A_{1}$ which $\varphi$ maps onto it.

Proposition. If $\varphi: A_{1} \rightarrow A_{2}$ is a continuous homomorphism from a complete metrizable group onto a polonais group $A_{2}$, then $\varphi$ is open iff there is a polonais subgroup $A_{1}^{\prime} \subset A_{1}$ such that $\varphi\left(A_{1}^{\prime}\right)=A_{2}$.

Proof. One direction is clear; if there exists such an $A_{1}^{\prime}, \varphi$, restricted to $A_{1}^{\prime}$, must be open, because the open mapping theorem for polonais groups applies, and consequently $\varphi$ must be open as well. Now suppose that $\varphi$ is open, and let $\Delta_{1}$ be a covering of $A_{1}$ by open spheres of radius one. The open sets $\varphi\left(\Delta_{1}\right)$ cover $A_{2}$ and since $A_{2}$ is Lindelöf, a countable subfamily also covers $A_{2}$. Throw away all members of $\Delta_{1}$ except for a countable number whose images cover $A_{2}$. For each member of this subcollection take a covering by open spheres of radius one-half and again throw away all but a countable number, and do this for $1 / n, n=3,4, \ldots$. Then the set of points in $A_{1}$ lying in the spheres that have been retained forms a separable space since it is Lindelöf, and $\varphi$ maps the closed subgroup generated by this set onto $\boldsymbol{A}_{2}$. To see this, let $a_{2}$ be in $\boldsymbol{A}_{2} ; a_{2}$ is in a sequence of open sets which are the images under $\varphi$ of open $1 / n$-spheres in $A_{1}$, the diameter of these sets tending to zero, from the continuity of $\varphi$. Because $A_{1}$ is complete, and because the sequence of $1 / n$-spheres mapping into the sequence of open sets containing $a_{2}$ is nested, their intersection contains just one point, which is in the closure of our separable set in $A_{1}$, and must map onto $a_{2}$. 
Definition. A continuous onto $G$-module map $\varphi: A_{1} \rightarrow A_{2}$ of complete metrizable groups is called amenable to $(G, X)$ if (1) every separable subset of $A_{2}$ is admissible and (2) if $S$ is a separable subset of $A_{2}$, there is a section $t$ for $\varphi \mid \varphi^{-1}(S)$ such that for $y$ in $Y,\left(A_{1}\right)_{s(y)} \rightarrow\left(A_{2}\right)_{s(y)}$ onto implies that $t\left(\left(A_{2}\right)_{s(y)} \cap S\right) \subset\left(A_{1}\right)_{s(y)}$.

Proposition. If $\varphi: A_{1} \rightarrow A_{2}$ is an onto continuous $G$-module map of complete metrizable groups, then if $\varphi_{*}$ maps $B\left(X, A_{1}\right)^{G}$ onto $B\left(X, A_{2}\right)^{G}$, it follows that for each $x$ in $X$, the map $\varphi_{x}:\left(A_{1}\right)_{x} \rightarrow\left(A_{2}\right)_{x}$ is onto. If further, $\varphi$ is amenable to $(G, X)$, then $\varphi_{s(y)}$ onto for each $y$ in $Y$ forces $\varphi_{*}$ to be onto.

Proof. If $a_{2}$ is in $\left(A_{2}\right)_{x}$, let $f$ in $B\left(X, A_{2}\right)^{G}$ be such that $f(x)=a_{2}$. Then for any $f^{\prime}$ in $B\left(X, A_{1}\right)^{G}$ with $\varphi_{*} f^{\prime}=f, f^{\prime}(x)$ is in $\left(A_{1}\right)_{x}$ and $\varphi_{x}\left(f^{\prime}(x)\right)=a_{2}$. For the second part, assume the amenability condition, let each $\varphi_{s(y)}$ be onto, and suppose that $f$ is in $B\left(X, A_{2}\right)^{G}$. If $t: S \rightarrow A_{1}$ is our assumed section for $S$ the range of $f$, consider $t \circ f \circ s$. This is a Borel function from $Y$ to $A_{1}$, and $t \circ f \circ s(y)$ is in $\left(A_{1}\right)_{s(y)}$ for $y$ in $Y$. The function we are after is none other than $\beta(t \circ f \circ s)$, since $\varphi_{*}(\beta(t \circ f \circ s))$ $=\beta \circ \varphi_{*}(t \circ f \circ s)=\beta(\varphi \circ t \circ f \circ s)=\beta(f \circ s)=\beta(\alpha(f))=f . S$ is separable; for this see $[10$, p. 305].

Now assume that $(G, X)$ is such that for the induced action $\left(G, X^{n}\right)$, there are Borel sections $s$ for $X^{n} \rightarrow X^{n} / G=Y_{n}$, and $u$ for $G \times s\left(Y_{n}\right) \rightarrow X^{n}$, and that $Y_{n}$ is polonais in the quotient topology. If these conditions are satisfied for all $n$, we say that the action $(G, X)$ is Borel smooth.

Proposition. If $0 \rightarrow A_{1} \stackrel{\alpha}{\rightarrow} A \stackrel{\oplus}{\rightarrow} A_{2} \rightarrow 0$ is an exact sequence of $G$-modules and $G$-module maps with $\alpha$ a homeomorphism into and $\varphi$ continuous and open, $\varphi$ amenable to the Borel smooth $(G, X)$, then if the maps $\varphi_{U}: A^{U} \rightarrow A_{2}^{U}$, where $U$ is $\bigcap_{i=1}^{n} G_{x_{i}}$, $x_{i}$ in $X$, are onto, we have a long exact sequence $\rightarrow H^{n-1}\left(X ; G, A_{2}\right) \rightarrow H^{n}\left(X ; G, A_{1}\right)$ $\rightarrow H^{n}(X ; \boldsymbol{G}, \boldsymbol{A}) \rightarrow H^{n}\left(\boldsymbol{X} ; \boldsymbol{G}, \boldsymbol{A}_{2}\right) \rightarrow H^{n+1}\left(X ; \boldsymbol{G}, \boldsymbol{A}_{1}\right) \rightarrow$, zero for $n<0$, and infinite to the right.

Proof. We apply the previous proposition to $B\left(X^{n}, A\right)^{G}$ and $B\left(X^{n}, A_{2}\right)^{G}$. Then $A_{x}=A^{U}$ for $x=\left(x_{1}, \ldots, x_{n}\right)$ in $X^{n}$, and we obtain an exact sequence $0 \rightarrow B^{n}\left(X ; G, A_{1}\right)$ $\rightarrow B^{n}(X ; G, A) \rightarrow B^{n}\left(X ; G, A_{2}\right) \rightarrow 0$ whose maps commute with the coboundary maps for all $n>0$, giving a map of complexes. The rest is algebraic and classical.

The condition that $\varphi_{U}: A^{U} \rightarrow A_{2}^{U}$ be onto can be rephrased homologically. For the group $U$ operating on itself by left translations, we have a long exact sequence for $0 \rightarrow A_{1} \rightarrow A \rightarrow A_{2} \rightarrow 0$. The first few terms of this sequence are $0 \rightarrow A_{1}^{U}$ $\rightarrow A^{U} \rightarrow A_{2}^{U} \rightarrow H^{1}\left(U, A_{1}\right) \rightarrow$, which shows that all we really must require is that the map $A_{2}^{U} \rightarrow H^{1}\left(U, A_{1}\right)$ be zero, and this is satisfied if $H^{1}\left(U, A_{1}\right)$ is zero.

Let us now determine precisely the image of $B\left(X, A_{1}\right)^{G}$ in $B\left(X, A_{2}\right)^{G}$ under the $\operatorname{map} \varphi_{*}$.

Proposition. Let $\varphi: A_{1} \rightarrow A_{2}$ be a continuous onto $G$-module map such that $A_{1}, A_{2}$ are complete metrizable and for every separable admissible subset $S \subset A_{2}$ 
there is a Borel section $t: S \rightarrow A_{1}$, and for all $y$ in $Y,\left(A_{1}\right)_{s(y)} \rightarrow\left(A_{2}\right)_{s(y)}$ onto implies $t\left(\left(A_{2}\right)_{s(y)} \cap S\right) \subset\left(A_{1}\right)_{s(y)}$. Then the image of $\varphi_{*}$ consists of those $f$ which have admissible range.

Proof. One way has been proved, and it suffices to remark that if $f$ is in $\boldsymbol{B}\left(\boldsymbol{X}, \boldsymbol{A}_{2}\right)^{\boldsymbol{G}}$ and $f^{\prime}$ is in $\boldsymbol{B}\left(\boldsymbol{X}, \boldsymbol{A}_{1}\right)^{G}$ and $\varphi_{*} f^{\prime}=f$, then since the range of $f^{\prime}$ is separable and maps onto the range of $f$ under $\varphi$, the range of $f$ is separable and admissible.

If $\boldsymbol{G}$ operates freely on $\boldsymbol{X}$, then $\boldsymbol{G}_{\boldsymbol{x}}=1$ for all $x$ in $\boldsymbol{X}$, and in this case there are no conditions on the Borel section $t$ except that it exist, which it always does in the above circumstances, and thus we get always a long exact sequence. If $G$ is also transitive we are almost in the classical case of Moore, [16, p. 43].

Suppose that we are given a morphism $(f, \alpha):(\boldsymbol{G}, \boldsymbol{X}) \rightarrow(\boldsymbol{H}, \boldsymbol{Y}), \alpha$ Borel, and a $G$-module $A_{2}$, an $H$-module $A_{1}$, and a map $\varphi: A_{1} \rightarrow A_{2}$ of $G$-modules, cunsidering $A_{1}$ as a $G$-module by means of the map $f$. Then there is induced a map $(f, \alpha, \varphi)_{*}^{n}$ from $B^{n}\left(\boldsymbol{Y} ; \boldsymbol{H}, \boldsymbol{A}_{1}\right)^{\boldsymbol{H}}$ to $\boldsymbol{B}^{n}\left(\boldsymbol{X} ; \boldsymbol{G}, \boldsymbol{A}_{2}\right)^{\boldsymbol{G}}$, defined as follows: $(f, \alpha, \varphi)_{*}^{n} h\left(x_{1}, \ldots, x_{n}\right)$ $=\varphi\left(h\left(\alpha\left(x_{1}\right), \ldots, \alpha\left(x_{n}\right)\right)\right)$, for $h$ in the group $B^{n}\left(\boldsymbol{Y} ; \boldsymbol{H}, \boldsymbol{A}_{1}\right)^{H}$, and this induced map commutes with the respective coboundary maps, thus inducing further maps $(f, \alpha, \varphi)_{*}^{n}$ on the cohomology groups $H^{n}\left(\boldsymbol{Y} ; \boldsymbol{H}, \boldsymbol{A}_{1}\right) \rightarrow H^{n}\left(\boldsymbol{X} ; \boldsymbol{G}, \boldsymbol{A}_{2}\right)$, called inflation maps. The property of these maps in which we are immediately interested is their functoriality, $\left(1_{G}, 1_{X}, 1_{A}\right)_{*}^{n}=1_{H^{n}(X ; G, A)},\left(1_{G}, \alpha \circ \beta, 1_{A}\right)_{*}^{n}=\left(1_{G}, \alpha, 1_{A}\right)_{*}^{n}$ $\circ\left(1_{G}, \beta, 1_{A}\right)_{*}^{n}$. The next proposition will serve to characterize those cohomology groups associated with a free $G$-action as those occurring in the classical action of $\boldsymbol{G}$ on itself by left translations.

Proposition. Let $\boldsymbol{G}$ act freely on $X$. Then for every $G$-module $A$ and all $n \geqq 0$, the inclusion $\left(1_{G}, i\right):(G, O) \rightarrow(G, X), O$ any orbit in $X$, induces onto isomorphisms $H^{n}(\boldsymbol{X} ; \boldsymbol{G}, \boldsymbol{A}) \rightarrow H^{n}(\boldsymbol{O} ; \boldsymbol{G}, \boldsymbol{A})$.

Proof. We shall define a $\boldsymbol{G}$-map $j: X \rightarrow O$ such that $j \circ i=1$ and $i^{*} \circ j^{*}=$ $1_{H^{n}(O ; G, A)}, j^{*} \circ i^{*}=1_{H^{n}(X ; G, A)}$. Toward this end, select a section $t$ for $\boldsymbol{G} \times s(Y) \rightarrow X$ and let $x_{0}=t_{2}\left(x_{0}\right)$ be that unique point of $\boldsymbol{O}$ such that $x_{0}=s\left(y_{0}\right)$ for some $y_{0}$ in $\boldsymbol{Y}$. Define $j(x)=j\left(t_{1}(x) t_{2}(x)\right)=t_{1}(x) x_{0}$. A straightforward calculation shows that $j \circ i=10$, and from functoriality, $i^{*} \circ j^{*}=1_{H^{n}(O ; G, A)}$ follows. If $i^{*}$ were one to one, we would be finished, since $i^{*} \circ\left(j^{*} \circ i^{*}\right)=\left(i^{*} \circ j^{*}\right) \circ i^{*}=1 \circ i^{*}=i^{*}$ implies that $j^{*} \circ i^{*}=1_{H^{n}(X ; G, A)}$. This will be shown if we show that every $f$ in $Z^{n}(X ; G, A)$ is cohomologous to an $f^{\prime}$ of the form $g \circ i$. If $f\left(x_{1}, \ldots, x_{k}, x_{k+1}, \ldots, x_{n}\right)$ is just $f\left(t_{1}\left(x_{1}\right) x_{0}, \ldots, t_{1}\left(x_{k}\right) x_{0}, x_{k+1}, \ldots, x_{n}\right)$, let $g\left(x_{1}, \ldots, x_{n-1}\right)$ be defined as

$$
f\left(x_{1}, \ldots, x_{k+1}, t_{1}\left(x_{k+1}\right) x_{0}, x_{k+1}, \ldots, x_{n}\right) \text {. }
$$

Then a long but simple-minded calculation shows that

$$
\begin{aligned}
& {\left[f-\partial\left((-1)^{k} g\right)\right]\left(x_{1}, \ldots, x_{k}, x_{k+1}, x_{k+2}, \ldots, x_{n}\right)} \\
& \quad=\left[f-\partial\left((-1)^{k} g\right)\right]\left(t_{1}\left(x_{1}\right) x_{0}, \ldots, t_{1}\left(x_{k}\right) x_{0}, t_{1}\left(x_{k+1}\right) x_{0}, x_{k+2}, \ldots, x_{n}\right) .
\end{aligned}
$$


This fact is used to perform an induction proof in the obvious manner, finishing the proof of the proposition.

\section{CHAPTER II. The Spectral Sequences}

In this chapter we obtain a spectral sequence given a closed normal subgroup $\boldsymbol{H}$ of a locally compact polonais group $\boldsymbol{G}$, for each of the four types of cohomology groups defined in the last chapter. The discrete theory will not be explicitly mentioned, since it is a special case of each of the other three. These reduce to the spectral sequences of Hochschild-Serre, Moore, and Mostow, as presented in [7], [16], and [17], respectively. Before we do this, we need a fact about Borel spaces of functions.

THEOREM. There is a natural injection $\theta: B(X, B(Y, A)) \rightarrow B(X \times Y, A)$ whose range consists of those $f$ which, when looked upon as functions from $X$ to $B(Y, A)$, have separable range.

Proof. For $f$ in $\boldsymbol{B}(\boldsymbol{X}, \boldsymbol{B}(\boldsymbol{Y}, \boldsymbol{A}))$ define $\theta f(x, y)=f(x)(y)$ and if $g$ is in $\boldsymbol{B}(\boldsymbol{X} \times \boldsymbol{Y}, \boldsymbol{A})$, let $\gamma g(x)(y)=g(x, y)$. Let $f$ be a Borel function from $X$ to $B(Y, A)$ of class $\alpha>0$, as defined on $[10$, p. 280]. Now because $f$ has separable range, we may apply (3), $\left[10\right.$, p. 294] to obtain the result that $f$ is the uniform limit of functions $f_{n}$ which are also of class $\alpha$, but are countably-valued. Now each $f_{n}$ is such that $\theta f_{n}$ is in $\boldsymbol{B}(\boldsymbol{X} \times \boldsymbol{Y}, \boldsymbol{A})$ and they converge pointwise to $\theta f$ in this space, showing that $\theta f$ is Borel. If $\alpha=0$, i.e., if $f$ is continuous, we just make use of a countable dense set in $\boldsymbol{X}$, which is a determining set for $f$, to see that $\theta f$ is Borel.

To show that the range of $\theta$ is as stated, let $f$ be in the group $B(X \times Y, A)$ and have separable range when considered as the function $\gamma f$. Now $\gamma f(x)$ is certainly in $B(Y, A)$ since the restriction of $f$ to $\{x\} \times Y$ is Borel. If $S$ is the separable range of $\gamma f$, if we can show that $(\gamma f)^{-1}\left(\left(W_{K, U}+h\right) \cap S\right)$ is Borel in $X$, we are finished. Set $W_{K, U}=\{f \mid f(K) \subset U, K$ compact, $U$ open containing 0$\}, h$ arbitrary in $S$. Because $S$ is separable every open set in $S$ is an at most countable union of these sets. Define $\hat{h}(x, y)=h(y)$. Then $\hat{h}$ is in $\boldsymbol{B}(\boldsymbol{X} \times \boldsymbol{Y}, \boldsymbol{A})$ and

$$
\begin{aligned}
(\gamma f)^{-1}\left(\left(W_{K, U}+h\right) \cap S\right) & =\left\{x \mid \gamma f(x) \in W_{K, U}+h\right\} \\
& =\{x \mid f(x, k)-h(k) \in U, \text { for } k \text { in } K\} \\
& =\{x \mid(f-\hat{h})(x, K) \subset U\} .
\end{aligned}
$$

Now since $f-\hat{h}$ is in $\boldsymbol{B}(\boldsymbol{X} \times \boldsymbol{Y}, \boldsymbol{A}),(f-\hat{h})^{-1}(U)$ is Borel in $\boldsymbol{X} \times \boldsymbol{Y}$, and all we really have to show is that if $B$ is Borel in $X \times Y, K$ compact in $Y$, then the set $\{x \mid(x, K) \subset B\}$ is Borel in $X$. If $B$ is open, this set is open. If now for all $\alpha<\beta<\Omega$, $\left\{x\right.$ in $X \mid(x, K)$ is in $\left.B, B \in B_{\alpha}\right\} \in B_{\alpha}$, where $B_{\alpha}$ is the $\alpha$ th Borel class of sets as on p. 251 of [10], we assume that $B$ is in $B_{\alpha}, B=\bigcup_{i=1}^{\infty} B^{i}, B^{i}$ in $B_{\alpha_{i}}, \alpha_{i}<\alpha$, we have $\{x$ in $X \mid(x, K) \subset B\}=\bigcup_{i=1}^{\infty}\left\{x\right.$ in $X \mid(x, K)$ in $\left.B^{i}\right\}$ is in $B_{\alpha}$, since $\left\{x\right.$ in $\left.X \mid(x, K) \subset B^{i}\right\}$ is in $B_{\alpha_{i}}$, and similarly if $B=\bigcap_{i=1}^{\infty} B^{i}, B^{i}$ in $B_{\alpha_{1}}, \alpha_{i}<\alpha$. This finishes the proof. 
Define $B^{p q}=B^{p+1}\left(G / H ; G / H, B^{q+1}(G ; H, A)^{H}\right)^{G / H}$, where $\boldsymbol{G} / \boldsymbol{H}$ operates on itself by left translations, $\boldsymbol{H}$ operates on $\boldsymbol{G}$ similarly, and we shorten this to

$$
B^{p+1}\left(G / H ; B^{q+1}(G ; H, A)^{H}\right)^{G / H} .
$$

Since $\boldsymbol{H}$ is normal, $\boldsymbol{G}$ leaves stable $\boldsymbol{B}^{a+1}(\boldsymbol{G} ; \boldsymbol{H}, \boldsymbol{A})^{\boldsymbol{H}}$ with the operation $\left(g_{1} f\right)(g)$ $=g_{1}\left(f\left(g_{1}^{-1} g\right)\right)$ and $H$, with the induced action, acts trivially, which gives an operation of $\boldsymbol{G} / \boldsymbol{H} . \boldsymbol{B}^{p q}$ has two coboundary maps $\delta_{1}^{p q}: B^{p q} \rightarrow B^{p+1, q}$ and $\delta_{2}^{p q}: B^{p q} \rightarrow B^{p, q+1}$ defined as

$$
\begin{aligned}
& \delta_{1} f\left(y_{1}, \ldots, y_{p+2}\right)\left(g_{1}, \ldots, g_{q+1}\right)=\sum_{k=1}^{p+2}(-1)^{k+1} f\left(y_{1}, \ldots, y_{k}, \ldots, y_{p+2}\right)\left(g_{1}, \ldots, g_{q+1}\right) \\
& \delta_{2} f\left(y_{1}, \ldots, y_{p+1}\right)\left(g_{1}, \ldots, g_{q+2}\right)=\sum_{k=1}^{q+2}(-1)^{k+1} f\left(y_{1}, \ldots, y_{p+1}\right)\left(g_{1}, \ldots, g_{k}, \ldots, g_{q+2}\right) .
\end{aligned}
$$

As in $\left[5\right.$, p. 86], there are two spectral sequences $\mathrm{I}_{r}^{p q}, \mathrm{II}_{r}^{p q}$ associated to this double complex, each of which converges to the cohomology of the total complex, appropriately filtered. To see what this cohomology is, let us look at $\mathrm{II}_{r}^{p q}$.

LEMMA. II ${ }_{r}^{p q}=0$ unless $p=0$.

Proof. $\mathrm{II}_{1}^{p q}$ is the cohomology of $\boldsymbol{B}^{p q}$ using $\delta_{1}$. But if $f$ is a cocycle in $\boldsymbol{B}^{p q}$ with respect to $\delta_{1}, p \neq 0$, and $\varphi$ the quotient map of $\boldsymbol{G}$ onto $\boldsymbol{G} / \boldsymbol{H}$, by defining $\alpha f\left(y_{1}, \ldots, y_{p}\right)\left(g_{1}, \ldots, g_{q+1}\right)$ to be $f\left(\varphi\left(g_{1}\right), y_{1}, \ldots, y_{p}\right)\left(g_{1}, \ldots, g_{q+1}\right)$, we see that $\alpha f$ is in $B^{p-1, q}$, since it has separable range and we have also $\delta_{1}(\alpha f)=f$.

COROLLARY. $\mathrm{II}_{2}^{0 q} \cong \mathrm{II}_{\infty}^{0 q} \cong$ the cohomology of $B^{q+1}(G, A)^{G}$ with $G$ as operators.

Proof.

$$
\begin{aligned}
\mathrm{II}_{1}^{0 q} & \cong H^{0}\left(\boldsymbol{G} / \boldsymbol{H}, \boldsymbol{B}^{q+1}(\boldsymbol{G} ; \boldsymbol{H}, \boldsymbol{A})^{H}\right) \\
& =\left(\left(B^{q+1}(\boldsymbol{G} ; \boldsymbol{H}, \boldsymbol{A})^{\boldsymbol{H}}\right)^{G / H} \cong B^{q+1}(G, A)^{G} .\right.
\end{aligned}
$$

The largest problem immediately facing us is that of formulating reasonable restrictions on $B^{q+1}(G ; H, A)$ which will allow us to identify $I_{1}^{p q}$ and $I_{2}^{p q}$ with something humanly recognizable. Now because the action of $\boldsymbol{H}$ on $\boldsymbol{G}$ by left translations is free, we have an isomorphism $H^{n}(G ; H, A) \cong H^{n}(H, A)$, since the orbits are just the left cosets of $\boldsymbol{H}$ in $\boldsymbol{G}$. The construction in the last chapter giving this isomorphism can be applied to the last $q+1$ variables of the complex $B^{p q}$ and we obtain an isomorphism between the spectral sequences of $B^{p q}$ and that of the following double complex, $B_{1}^{p q}=B^{p+1}\left(G / H, B^{q+1}(H, A)^{H}\right)^{G / H}$; in fact, there are isomorphisms $\mathrm{I}_{1}^{p q} \cong\left(\mathrm{I}_{2}^{p q}\right)_{1}$ for all $p, q \geqq 0$, commuting with the respective $\delta_{2}$ 's. Henceforth we shall work exclusively with the double complex $B_{1}^{p q}$ and perversely denote it by $\boldsymbol{B}^{\text {pq }}$.

The cocycles of $B^{p q}$ with respect to $\delta_{2}$ are just those $f$ in $B^{p q}$ which take values in $Z^{q+1}(H, A)$. Identifying the coboundaries is a little harder. If $\partial\left(B^{q}(H, A)^{H}\right)$ is closed, and if we factor $\partial: B^{q}(H, A)^{H} \rightarrow \partial\left(B^{q}(H, A)^{H}\right)$ as

$$
B^{\alpha}(H, A)^{H} \stackrel{\alpha}{\longrightarrow} B^{q}(H, A)^{H} / Z^{\alpha}(H, A) \stackrel{\beta}{\longrightarrow} \partial\left(B^{\alpha}(H, A)^{H}\right)
$$


where $\alpha$ is the continuous open quotient map and $\beta$ is a continuous one to one map, a separable subset of $\partial\left(B^{q}(\boldsymbol{H}, \boldsymbol{A})^{\boldsymbol{H}}\right)$ is admissible for $\partial$ iff it is admissible for $\beta$, since every separable subset of the above quotient is admissible for $\alpha$. We can apply an earlier criterion to $\partial$ to discover that the image of the map $\delta_{1}$ taking $B^{p+1}\left(G / H, B^{q}(H, A)^{H}\right)^{G / H}$ into $B^{p+1}\left(G / H, \partial\left(B^{q}(H, A)^{H}\right)\right)^{G / H}$ is just the set of $f$ in the latter group having admissible range.

Definition. $B^{p q}$ is called full if $\partial$ is amenable to $\left(\boldsymbol{G} / \boldsymbol{H},(\boldsymbol{G} / \boldsymbol{H})^{p+1}\right)$.

In case $B^{p q}$ is full, this implies, in particular, that the range of $\partial$ is closed, since amenability was defined only for complete groups; also we may identify the coboundaries with respect to $\delta_{2}$ as those $f$ in $B^{p q}$ taking values in $\partial\left(B^{q}(H, A)^{H}\right)$. The reason that the previous theorems concerning ontoness of induced maps are applicable is that all the actions $\left(\boldsymbol{G} / \boldsymbol{H},(\boldsymbol{G} / \boldsymbol{H})^{p+1}\right)$ have the proper Borel sections. Indeed, the action of $\boldsymbol{G} / \boldsymbol{H}$ on $(\boldsymbol{G} / \boldsymbol{H})^{p+1}$ is that by left translation by members of the diagonal subgroup corresponding to $\boldsymbol{G} / \boldsymbol{H}$; similarly for $\left(\boldsymbol{H}, \boldsymbol{H}^{q+1}\right)$. Because every continuous function is Borel, there is an injection $Z_{c}^{n}(H, A) \rightarrow Z^{n}(H, A)$, which takes coboundaries into coboundaries, thus inducing a natural map on cohomology $H_{c}^{n-1}(\boldsymbol{H}, \boldsymbol{A}) \rightarrow H^{n-1}(\boldsymbol{H}, \boldsymbol{A})$.

LEMMA. If the natural homomorphism above is onto for $n-1=q$, with $B^{p q}$ full, and the groups are Hausdorff, then $Z^{q+1}(H, A) \rightarrow H^{q}(H, A)$ is amenable to $\left(\boldsymbol{G} / \boldsymbol{H},(\boldsymbol{G} / \boldsymbol{H})^{p+1}\right)$.

Proof. As $H_{c}^{q}$ is separable and $H_{c}^{q} \rightarrow H^{q}$ is continuous, $H^{q}$ is also separable. $H_{c}^{q} \rightarrow H^{q}$ is open as well, from the open mapping theorem. We have the following commutative diagram with exact rows and columns:

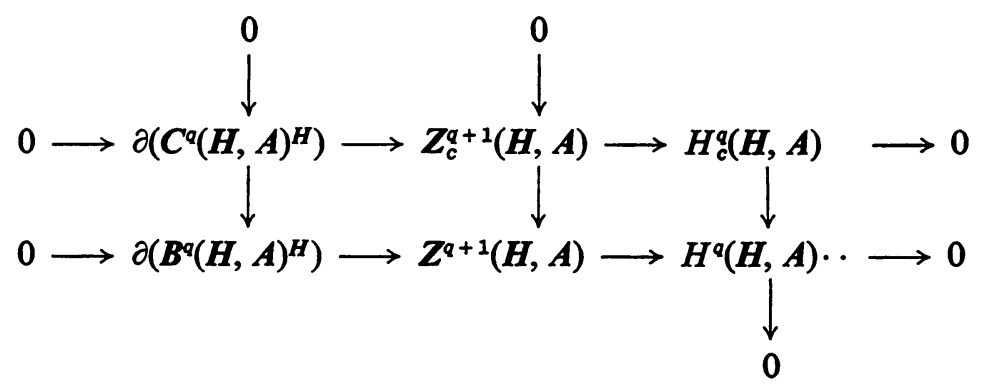

where the dotted arrow has been filled in from the information in the rest of the diagram. This gives the desired amenability.

COROLLARY. Under the above hypotheses, there is an isomorphism

$$
\mathrm{I}_{2}^{p q} \cong H^{p}\left(\boldsymbol{G} / \boldsymbol{H}, H^{q}(\boldsymbol{H}, \boldsymbol{A})\right)
$$


Proof. The diagram

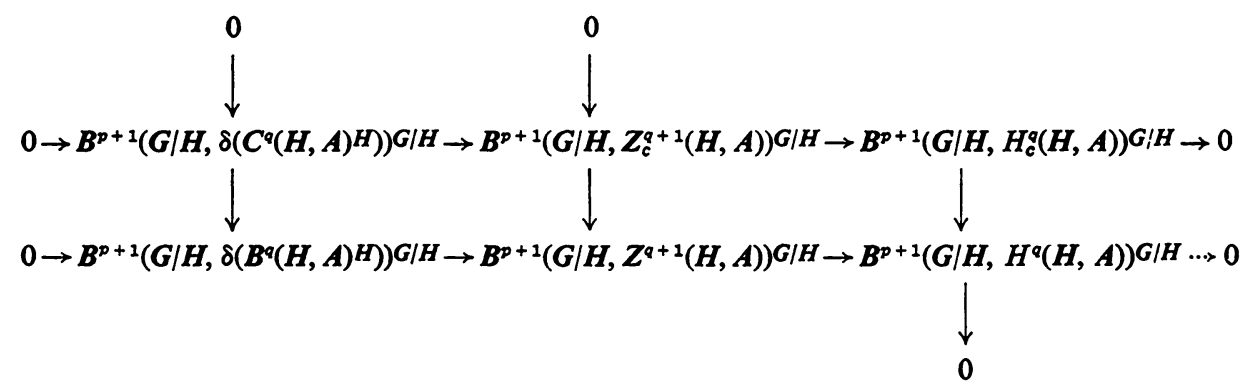

commutative and with exact rows and columns, where the dotted arrow has been filled in homologically, just says that $B^{p+1}\left(G / H, H^{q}(H, A)\right)^{G / H} \cong I_{1}^{p q}$, and as this isomorphism commutes with $\delta_{1}$, the above isomorphism results.

Corollary. If $H^{1}(\boldsymbol{H}, \boldsymbol{A})$ is Hausdorff and if $\boldsymbol{A}$ is polonais,

$$
\mathrm{I}_{2}^{p 1} \cong H^{p}\left(\boldsymbol{G} / \boldsymbol{H}, H^{1}(\boldsymbol{H}, \boldsymbol{A})\right) .
$$

Proof. Using a variant of Banach's homomorphism theorem, it follows easily that $\boldsymbol{Z}_{c}^{2}(\boldsymbol{H}, \boldsymbol{A})=\boldsymbol{Z}^{2}(\boldsymbol{H}, \boldsymbol{A})$, and that $\partial\left(C^{1}(\boldsymbol{H}, \boldsymbol{A})^{H}\right)$ is $\partial\left(B^{1}(\boldsymbol{H}, \boldsymbol{A})^{H}\right)$; hence in particular, $H_{c}^{1}(\boldsymbol{H}, \boldsymbol{A}) \cong H^{1}(\boldsymbol{H}, \boldsymbol{A}) . \boldsymbol{B}^{p 1}$ is full since the map $\boldsymbol{B}^{1}(\boldsymbol{H}, \boldsymbol{A})^{H} \rightarrow \partial\left(\boldsymbol{B}^{1}(\boldsymbol{H}, \boldsymbol{A})^{\boldsymbol{H}}\right)$ is just $A \rightarrow A / A^{H}$, the quotient map, if $A$ is polonais. This corollary is just Theorem 1.1 of [16].

COROLlaRY. If $\boldsymbol{H}$ is discrete and $\boldsymbol{A}$ is compact, then for all $p, q$,

$$
\mathrm{I}_{2}^{p q} \cong H^{p}\left(\boldsymbol{G} / \boldsymbol{H}, H^{q}(\boldsymbol{H}, \boldsymbol{A})\right) .
$$

Proof. The groups $B^{q+1}(H, A)$ are all compact and so the groups $\partial\left(B^{q+1}(H, A)^{H}\right)$ are all closed and $\partial$ is open onto its image, showing its amenability to $\left(\boldsymbol{G} / \boldsymbol{H},(\boldsymbol{G} / \boldsymbol{H})^{p+1}\right)$ for all $q$. The last word is $H_{c}^{q}(\boldsymbol{H}, \boldsymbol{A})=H^{q}(\boldsymbol{H}, \boldsymbol{A})$.

COROLlaRY. If $\boldsymbol{H}$ is open, $\mathrm{I}_{2}^{p q} \cong H^{p}\left(\boldsymbol{G} / \boldsymbol{H}, H^{q}(\boldsymbol{H}, \boldsymbol{A})\right)$ for all $p, q$.

Proof. Left to the reader.

We now turn to the continuous cohomology groups. Call the action $(G, X)$ continuously regular if (1) there is a continuous section $s$ for the quotient map $\varphi: X \rightarrow X / G=Y$ and (2) there is further a continuous section for the restriction of $\boldsymbol{G} \times \boldsymbol{X} \rightarrow \boldsymbol{X}$ to $\boldsymbol{G} \times \boldsymbol{s}(\boldsymbol{Y})$. Then the following lemmas can be proved, using techniques similar to those used to prove the corresponding lemmas in the Borel case.

LEMMA. If $(\boldsymbol{G}, \boldsymbol{X})$ is continuously regular, there is an isomorphism between the group of continuous $f: \boldsymbol{Y} \rightarrow \boldsymbol{A}$ such that for $y$ in $\boldsymbol{Y}, f(y)$ is in $\check{\boldsymbol{A}_{s(y)}}$ and $\boldsymbol{C}(\boldsymbol{X} ; \boldsymbol{G}, \boldsymbol{A})^{\boldsymbol{G}}$, where $\check{A}_{s(y)}$ is the set $\left\{a \in A_{s(y)} \mid\right.$ there is $f$ in $C(X ; G, A)^{G}$ and $\left.f(x)=a\right\}$.

Definition. An onto $G$-module map $\varphi: A_{1} \rightarrow A_{2}$ is called continuously amenable to $(G, X)$ if $\varphi$ is amenable to $(G, X)$ and in addition, the section $t: A_{2} \rightarrow A_{1}$ can be chosen continuous. 
In the particular case that $\check{A}_{s(y)}$ is open in $A$ for all $y$ in $Y$, the set of $y$ in $Y$ such that for all $f: Y \rightarrow A$ satisfying the above conditions, $f(y)$ is in $\check{A_{s\left(y_{0}\right)}}$, for some

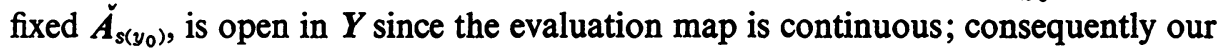
group of functions on $Y$ is isomorphic to $\pi C\left(Y_{\alpha}, A_{\alpha}\right)(\alpha \in D), D$ a set indexing the partition.

THEOREM. If $\varphi: A_{1} \rightarrow A_{2}$ is a continuous onto G-module map and if

$$
\varphi_{*}: C\left(X ; G, A_{1}\right)^{G} \rightarrow C\left(X ; G, A_{2}\right)^{G}
$$

is onto, then $\varphi_{x}:\left(A_{1}\right)_{x} \rightarrow\left(A_{2}\right)_{x}$ is onto for each $x$ in $X$. If $\varphi$ is continuously amenable to the continuously regular $(G, X)$, then $\varphi_{s(y)}$ onto for all $y$ in $Y$ forces $\varphi_{*}$ to be onto.

Proposition. If $0 \rightarrow A_{1} \stackrel{\alpha}{\rightarrow} A \stackrel{\beta}{\rightarrow} A_{2} \rightarrow 0$ is an exact sequence of $G$-modules, and if $\alpha$ is a homeomorphism, $\varphi$ continuous, open and admitting a continuous section making $\varphi$ amenable to the continuously regular $(G, X)$, and if $\varphi_{U}: A^{U} \rightarrow A_{2}^{U}$ are onto, there is a long exact sequence for the continuous groups.

Proposition. Let $\boldsymbol{G}$ act freely on $\boldsymbol{X}$ with $(G, X)$ continuously regular.

Then $(G, O) \rightarrow(G, X)$ induces isomorphisms

$$
H_{c}^{n}(\boldsymbol{X} ; \boldsymbol{G}, \boldsymbol{A}) \rightarrow H_{c}^{n}(\boldsymbol{O} ; \boldsymbol{G}, \boldsymbol{A}), \text { for all } n \text {. }
$$

Definition. A closed subgroup $\boldsymbol{H}$ of a topological group $\boldsymbol{G}$ is said to be continuously embedded in $G$ if there is a continuous section for the quotient map $\boldsymbol{G} \rightarrow \boldsymbol{G} / \boldsymbol{H}$.

LEMMA. The property of being closed and continuously embedded is transitive.

Proof. Let $\varphi_{1}: H_{3} \rightarrow H_{3} / H_{1}, \varphi_{2}: H_{3} \rightarrow H_{3} / H_{2}, \varphi_{3}: H_{3} / H_{1} \rightarrow H_{3} / H_{2}, \varphi_{4}: H_{2}$ $\rightarrow H_{2} / H_{1}$, and $s_{2}, s_{4}$ their respective continuous sections, where $H_{1} \subset H_{2} \subset H_{3}$ are closed subgroups. $s_{3}=\varphi_{1} \circ s_{2}$ is a continuous section for $\varphi_{3}$, and the section for which we are searching is defined for $x$ in $H_{3} / H_{1}$ as

$$
s_{1}(x)=\left[s_{2} \circ \varphi_{3}(x)\right] s_{4}\left(\left[s_{2} \circ \varphi_{3}(x)\right]^{-1} x\right) .
$$

Now we move on to the business of constructing a spectral sequence for the continuous cohomology groups. There are more sophisticated devices available, but for our purposes, it suffices to assume continuous sections for practically every map in sight, in order to identify $I_{2}^{p q}$. See $[17$, p. 27] for some ideas along this line.

Consider the following subcomplex of $B^{p q}$ :

$$
C^{p q}=C^{p+1}\left(G / H, C^{q+1}(G ; H, A)^{H}\right)^{G / H}
$$

with the two coboundary maps $\delta_{1}, \delta_{2}$ restricted. Then $\delta_{1}^{p q}: C^{p q} \rightarrow C^{p+1, q}$ and $\delta_{2}^{p q}: C^{p q} \rightarrow C^{p, q+1}$. We then have two spectral sequences I, II, both of which converge to the cohomology of the total complex, which is $\mathrm{II}_{2}^{0 q} \cong \mathrm{II}_{\infty}^{0 q} \cong H_{c}^{q}(G, A)$. If the closed normal subgroup $\boldsymbol{H}$ is embedded continuously in the locally compact 
group $\boldsymbol{G}$, it follows from the transitivity proved above, that the image of $\boldsymbol{H}$ in the diagonal subgroup of $G^{q+1}$ is embedded continuously in $G^{q+1}$ for all $q$, since the diagonal subgroup $\boldsymbol{G}$ of $\boldsymbol{G}^{q+1}$ is continuously embedded.

THEOREM. If we have the following continuous embeddings: $\boldsymbol{H} \subset \boldsymbol{G}$,

$$
\partial\left(\left(C^{q}\right)^{H}\right) \subset Z_{c}^{q+1}, \quad Z_{c}^{q} \subset\left(C^{q}\right)^{H},
$$

then $\mathrm{I}_{2}^{p q} \cong H_{c}^{p}\left(\boldsymbol{G} / \boldsymbol{H}, H_{c}^{q}(\boldsymbol{H}, \boldsymbol{A})\right)$.

Proof. If $s$ is a continuous section for $\boldsymbol{G}^{q+1} \rightarrow \boldsymbol{G}^{q+1} / \boldsymbol{H}$, where $\boldsymbol{H}$ is embedded in the diagonal subgroup continuously, a section for $H \times s\left(G^{q+1} / H\right) \rightarrow G^{q+1}$ is given by $x \rightarrow\left(x[s \circ \varphi(x)]^{-1}, s \circ \varphi(x)\right)$ and is a homeomorphism. Since $H$ is normal, closed, and continuously embedded in $\boldsymbol{G}$, and acts freely on $\boldsymbol{G}$ by left translations, a construction as in the Borel case shows that the isomorphism

$$
H_{c}^{q}(\boldsymbol{H}, \boldsymbol{A}) \cong H_{c}^{q}(\boldsymbol{G} ; \boldsymbol{H}, \boldsymbol{A})
$$

gives. rise to an isomorphism $\mathrm{I}_{1}^{p q} \cong$ cohomology of $C^{p q}$ using $\delta_{2} \cong$ cohomology of $C^{p+1}\left(G / H, C^{q+1}(H, A)^{H}\right)^{G / H}$ using $\delta_{2}$, which commutes with the respective maps $\delta_{1}$. Now the assumed continuous embeddings allow us to show the following sequence exact:

$$
\begin{aligned}
0 & \rightarrow C^{p+1}\left(G / H, \partial\left(C^{q}(H, A)^{H}\right)\right)^{G / H} \\
& \rightarrow C^{p+1}\left(G / H, Z_{c}^{q+1}(H, A)\right)^{G / H} \\
& \rightarrow C^{p+1}\left(G / H, H_{c}^{q}(H, A)\right)^{G / H} \rightarrow 0 .
\end{aligned}
$$

But the first and second terms of this sequence are just the coboundaries and cocycles of $C^{p q}$ with respect to $\delta_{2}$, giving $\mathrm{I}_{1}^{p q} \cong C^{p+1}\left(G / H, H_{c}^{q}(H, A)\right)^{G / H}$, this isomorphism commuting with $\delta_{1}$; thus $I_{2}^{p q} \cong H_{c}^{p}\left(G / H, H_{c}^{q}(\boldsymbol{H}, A)\right.$ ), as claimed.

Now enter the most delicate of the three cohomology theories: the bounded Borel theory. The conditions to be imposed on the maps occurring are satisfied in quite a number of interesting and useful cases. The main reason for giving this theory any attention at all is that it is possible to prove the isomorphism of the continuous groups with the bounded Borel in many more instances than with the Borel. We remark here that if $\boldsymbol{H}$ is a closed subgroup of a locally compact group $\boldsymbol{G}$, then there is a bounded Borel section for the quotient map, as shown in Mackey [12, Lemma 1.1]. We list without proof the appropriate lemmas and definitions for this theory.

Defintion. $(G, X)$ is called bounded if there is a bounded Borel section for $X \rightarrow X / G=Y$ and a section for

$$
\boldsymbol{G} \times s(\boldsymbol{Y}) \rightarrow \boldsymbol{X} .
$$

LEMMA. For $(G, X)$ bounded, there is an isomorphism between $\bar{B}(X, A)^{G}$ and the group of bounded Borel functions ffrom $Y$ to $A$ such that $f(y)$ is in $A_{s(y)}$ for all $y$ in $Y$. 
Definition. $\varphi: A_{1} \rightarrow A_{2}$ is boundedly amenable to $(G, X)$ if it is amenable and the section $t$ can be chosen bounded.

Proposition. $\varphi: A_{1} \rightarrow A_{2}$ onto implies that if $\varphi_{*}: \bar{B}\left(X, A_{1}\right)^{G} \rightarrow \bar{B}\left(X, A_{2}\right)^{G}$ is onto, then $\varphi_{x}$ is onto for each $x$ in $X$. If $\varphi$ is boundedly amenable to the bounded $(G, X)$, then $\varphi_{s(y)}$ onto for all $y$ in $Y$ gives us that $\varphi_{*}$ is onto.

Proposition. If $0 \rightarrow A_{1} \rightarrow A \rightarrow A_{2} \rightarrow 0$ is an exact sequence of complete metrizable $\boldsymbol{G}$-modules, with $\varphi$ boundedly amenable to the bounded action $\left(G, X^{n}\right)$ for all $n$, there is a long exact sequence involving the bounded groups if the $\varphi_{U}$ are all onto.

The double complex to consider is $\bar{B}^{p q}=\bar{B}^{p+1}\left(\boldsymbol{G} / \boldsymbol{H}, \bar{B}^{q+1}(\boldsymbol{G} ; \boldsymbol{H}, \boldsymbol{A})^{\boldsymbol{H}}\right)^{\boldsymbol{G} / \boldsymbol{H}}$ and the usual two coboundary maps $\delta_{1}, \delta_{2}$. There are also $\overline{\mathrm{I}}, \overline{\mathrm{II}}$, around which converge to the same limit, that of the total cohomology of $\bar{B}^{p q}$.

LEMMA. $\overline{\mathrm{I}}_{r}^{p q}=0$ unless $p=0$.

Proof. Define, as before, $\alpha f\left(y_{1}, \ldots, y_{p}\right)\left(g_{1}, \ldots, g_{q+1}\right)$ to be $f\left(\varphi\left(g_{1}\right), y_{1}, \ldots, y_{p}\right)$ $\cdot\left(g_{1}, \ldots, g_{q+1}\right)$ and let $K$ be compact in $G$; if we show that $f\left(K, y_{1}, \ldots, y_{p}\right)(K, \ldots, K)$ is precompact for fixed $\left(y_{1}, \ldots, y_{p}\right)$, this will be more than sufficient to show that $\alpha f:(\boldsymbol{G} / \boldsymbol{H})^{p} \rightarrow \overline{\boldsymbol{B}}^{q+1}(\boldsymbol{G} ; \boldsymbol{H}, \boldsymbol{A})^{\boldsymbol{H}}$. But since $f$ is in $\overline{\boldsymbol{B}}^{p q}$ and since a precompact set of functions must be uniformly bounded on every compact set, this must be a precompact set. To show that $\alpha f$ is bounded, let $\boldsymbol{C}$ be compact in $\boldsymbol{G} / \boldsymbol{H}$; we must show that the set of functions given by $f\left(\varphi\left(g_{1}\right), C, \ldots, C\right)\left(g_{1}, \ldots, g_{q+1}\right)$ is precompact in $\bar{B}^{q+1}(G ; \boldsymbol{H}, \boldsymbol{A})^{\boldsymbol{H}}$. Now a set of functions is precompact iff it is precompact on every compact subset of $G^{q+1}$. Let $K$ be compact in $G$; then the set of restrictions of the above family to $K^{q+1}$ is contained in the set $f(\varphi(K), C, \ldots, C)(K, \ldots, K)$ and this is precompact since $\varphi(K) \times C \times \cdots \times C$ is compact in $(\boldsymbol{G} / \boldsymbol{H})^{p+1}$, because $f$ takes this compact set into a precompact set of functions. This shows that $\alpha f$ is in $\bar{B}^{p-1, q}$, and it is straightforward to verify that $\delta_{1}(\alpha f)=f$.

COROLlaRY. $\mathrm{II}_{2}^{0 q} \cong \mathrm{II}_{\infty}^{0 q} \cong \bar{H}^{a}(G, A)$.

LEMMA. If $(\boldsymbol{G}, \boldsymbol{X})$ is bounded and $\boldsymbol{G}$ acts freely on $\boldsymbol{X}$, and $\boldsymbol{O}$ is any orbit, there is an isomorphism $\bar{H}^{n}(X ; G, A) \cong \bar{H}^{n}(O ; G, A)$.

COROllary. The injection of the double complex $\bar{B}^{p+1}\left(\boldsymbol{G} / \boldsymbol{H}, \bar{B}^{q+1}(\boldsymbol{H}, \boldsymbol{A})^{H}\right)^{G / H}$ into $\bar{B}^{p q}$ induces an isomorphism in the usual manner.

Definition. $\bar{B}^{p q}$ is full if $\partial: \bar{B}^{q}(\boldsymbol{H}, \boldsymbol{A})^{\boldsymbol{H}} \rightarrow \partial\left(\overline{\boldsymbol{B}}^{q}(\boldsymbol{H}, \boldsymbol{A})^{\boldsymbol{H}}\right)$ is boundedly amenable to $\left(\boldsymbol{G} / \boldsymbol{H},(\boldsymbol{G} / \boldsymbol{H})^{p+1}\right)$.

Notice that all of the actions $\left(\boldsymbol{H}, \boldsymbol{H}^{q+1}\right)$, and $\left(\boldsymbol{G} / \boldsymbol{H},(\boldsymbol{G} / \boldsymbol{H})^{p+1}\right)$ are bounded.

Proposition. If $\overline{\boldsymbol{B}}^{p q}$ is full and $H_{c}^{q}(\boldsymbol{H}, \boldsymbol{A}) \rightarrow \bar{H}^{q}(\boldsymbol{H}, \boldsymbol{A})$ is an onto continuous map of Hausdorff groups such that either (a) $\bar{H}^{a}(H, A)$ is locally compact or (b) the maps $H_{c}^{q}(\boldsymbol{H}, \boldsymbol{A}) \rightarrow \bar{H}^{q}(\boldsymbol{H}, \boldsymbol{A}), Z_{c}^{q+1}(\boldsymbol{H}, \boldsymbol{A}) \rightarrow H_{c}^{q}(\boldsymbol{H}, \boldsymbol{A})$ admit bounded Borel sections, then $\bar{I}_{2}^{p q}=\bar{H}^{p}\left(\boldsymbol{G} / \boldsymbol{H}, \bar{H}^{q}(\boldsymbol{H}, \boldsymbol{A})\right)$. 
COROLlary. If $\bar{H}^{1}(\boldsymbol{H}, \boldsymbol{A})$ is Hausdorff and $\boldsymbol{A}$ is locally compact, then for all $p$, $\overline{\boldsymbol{I}}_{2}^{p 1}=\bar{H}^{p}\left(\boldsymbol{G} / \boldsymbol{H}, \bar{H}^{1}(\boldsymbol{H}, \boldsymbol{A})\right)$.

Proof. $A \rightarrow A / A^{H}$ admits a bounded Borel section; indeed all we need assume is that $A / A^{H}$ is locally compact, which will be true if $\bar{Z}^{2}(H, A)$ is.

COROLLARY. If $\boldsymbol{H}$ is discrete and $\boldsymbol{A}$ compact, then for all $p, q$,

$$
\overline{\mathrm{I}}_{2}^{p q}=\bar{H}^{p}\left(\boldsymbol{G} / \boldsymbol{H}, \bar{H}^{q}(\boldsymbol{H}, \boldsymbol{A})\right) .
$$

COROLLARY. If $\boldsymbol{H}$ is open, we also get the above isomorphism.

In the next chapter, we pursue the question of relating the various cohomology theories and obtaining certain conditions for $\boldsymbol{B}^{p q}$ to be full.

\section{ChaPter III. The Computations}

The main tools having been collected and honed, it is now time to compute. Moore in [16] has done an admirable job of this for the second cohomology groups of a respectably large class of both domain groups $\boldsymbol{G}$ and coefficient groups $A$. In case $\boldsymbol{A} \cong T$, the circle group, and $G$ abelian operates trivially, Kleppner in [9] has determined $H^{2}(G, T)$, modulo certain 2-primary groups $G$, as the group of continuous bihomomorphisms on $G$ with values in $T$, modulo the symmetric ones. Motivated by the fact that in case $A \cong R$, the real line, $H^{2}(G, R) \cong \bigwedge^{2}(G, R)$, the group of alternating bihomomorphisms, $\boldsymbol{G}$ abelian operating trivially, we are able to show that this isomorphism is valid for all dimensions, and further that the cohomology of locally compact abelian groups with values in the reals is equivalent to the cohomology of discrete torsion-free groups with values in the reals. In doing this, we move freely between the homogeneous and the nonhomogeneous theories, as utility directs. Thus far we have worked solely with the homogeneous theory for two reasons: one, the nonhomogeneous theory is not at all applicable to the situation of a general group action $(\boldsymbol{G}, \boldsymbol{X})$, and two, the homogeneous theory is much more cleancut, and hence clearly suited to our tastes.

To pass from the homogeneous to the nonhomogeneous theory, define $\alpha: B^{n}(G, A)^{G} \rightarrow B^{n-1}(G, A)$, for $n \geqq 2$, as

$$
\alpha f\left(x_{1}, \ldots, x_{n-1}\right)=f\left(1, x_{1}, x_{1} x_{2}, \ldots, x_{1} \cdots x_{n-1}\right) .
$$

The inverse of $\alpha$ is $\beta$, which is

$$
f\left(x_{1}, \ldots, x_{n}\right)=x_{1} f\left(x_{1}^{-1} x_{2}, x_{2}^{-1} x_{3}, \ldots, x_{n-1}^{-1} x_{n}\right) .
$$

If we require this be a map of complexes, the coboundary operator on $B^{n-1}(G, A)$ must be

$$
\begin{aligned}
\partial f\left(x_{1}, \ldots, x_{n}\right)= & x_{1} f\left(x_{2}, \ldots, x_{n}\right)+\sum_{k=1}^{n-1}(-1)^{k} f\left(x_{1}, \ldots, x_{k} x_{k+1}, \ldots, x_{n}\right) \\
& +(-1)^{n} f\left(x_{1}, \ldots, x_{n-1}\right) .
\end{aligned}
$$


$\alpha$ is a topological isomorphism which is natural in all possible senses; in $G, A$, and the action of $\boldsymbol{G}$ on $\boldsymbol{A}$. There is a spectral sequence for this theory, which follows as a result of this strong isomorphism, and its properties parallel those of the homogeneous theory, as may be seen in [16], [17]. Let us be about our stated business.

LEMMA. If either $\boldsymbol{X}$ or $\boldsymbol{Y}$ is discrete, $\bar{B}(\boldsymbol{X}, \overline{\boldsymbol{B}}(\boldsymbol{Y}, \boldsymbol{A}))$ is isomorphic to $\overline{\boldsymbol{B}}(\boldsymbol{X} \times \boldsymbol{Y}, \boldsymbol{A})$.

Proof. $\bar{B}(\boldsymbol{Y}, \boldsymbol{A})$ is separable if $\boldsymbol{Y}$ is discrete, and every Borel function $f$ in $B(X, B(Y, A))$ has separable range.

LEMMA. A Borel function $f: G^{n} \rightarrow A$, which is a homomorphism in each variable separately, is continuous.

Proof. The lemma is actually valid for any polonais $\boldsymbol{G}$ and any complete metrizable $A$. We prove the lemma for $n=3$, the generalization to the case of any $n$ being both trivial and tedious. Let $f$ be as hypothesized, mapping $G^{3}$ to $A$. Suppose that $\{x(n)\}$ is a sequence approaching $x(0)$. Then by $[10, \mathrm{p} .306]$ there is a set $P$ of the first category such that $f$, restricted to the complement, is continuous. The set $S=\bigcup_{n \geqq 0} x(n)^{-1} P$ is also of the first category, and so there is a point $\left(g_{1}, g_{2}, g_{3}\right)$ not in $S$. Now expand $f\left(g_{1} x(n)_{1}, g_{2} x(n)_{2}, g_{3} x(n)_{3}\right)=f\left(x(n)_{1}, x(n)_{2}, x(n)_{3}\right)+$ terms which vary in only two places. This lets us express the first term on the right-hand side in terms of the others, the other functions approaching a limit, where we assume that we have already proved the theorem for $n=2$.

LEMMA. $H^{n}\left(Z^{m}, A\right) \cong \bigwedge^{n}\left(Z^{m}, A\right), Z^{m}$ acting trivially on $A$.

Proof. Take a Koszul resolution of $Z$ as a $Z\left[Z^{m}\right]$-module, as in [14, p. 204]. Then it follows easily that $H^{n}\left(Z^{m}, A\right) \cong \operatorname{Ext}_{\left[Z^{m}\right]}^{n_{3}}(Z, A)=\operatorname{Hom}_{Z}\left(E_{n}, A\right)$, where $E_{n}$ is the $n$th exterior algebra on the generators $u_{1}, \ldots, u_{m}$, corresponding to the generators of $Z^{m}$; but this last group is just $\bigwedge^{n}\left(Z^{m}, A\right)$.

Definition. If $A$ is divisible and torsion-free, then the alternation map $A: F^{n}(G, A)$ $\rightarrow F^{n}(G, A)$ is given by $A f\left(x_{1}, \ldots, x_{n}\right)=1 / n !\left(\sum(-1)^{\operatorname{ssn}(p)} f\left(x_{p(1)}, \ldots, x_{p(n)}\right)\right), p$ in $S_{n}$, the symmetric group.

LEMMA. If $A$ is torsion-free and divisible, there is an exact sequence

$$
0 \longrightarrow \partial\left(B^{n}\left(Z^{m}, A\right)\right) \stackrel{i}{\longrightarrow} Z^{n+1}\left(Z^{m}, A\right) \stackrel{A}{\longrightarrow} \bigwedge^{n+1}\left(Z^{m}, A\right) \longrightarrow 0,
$$

$i$ the inclusion map and $A$ the alternation.

Proof. It is straightforward to compute that $A(\partial g)=0$, for $g$ in $B^{n}(G, A)$, for any $G, A$. Also, from $[1$, p. 72$]$, it follows that $A\left(Z^{n+1}\left(Z^{m}, A\right)\right) \subset \bigwedge^{n+1}\left(Z^{m}, A\right)$. If $f$ is in $\wedge^{n+1}\left(Z^{m}, A\right)$, then $A f=f$, since $A^{2}=A$, showing that $A$ is onto. Now suppose that $f$ is in $Z^{n+1}\left(Z^{m}, A\right)$ and $A f=0$. From the previous lemma, write $f$ as $\partial g+h$, where $h$ is in $\bigwedge^{n+1}\left(Z^{m}, A\right)$; then since $A(\partial g)=0$ and $A h=h$, we get $h=A h=0$, and $f=\partial g$. 
THEOREM. If $\boldsymbol{A}$ is divisible and torsion-free, then $\partial\left(\boldsymbol{B}^{n}\left(\boldsymbol{Z}^{m}, \boldsymbol{A}\right)\right)$ is closed in $Z^{n+1}\left(Z^{m}, A\right)$. If $A$ is also polonais, then $\partial$ is open onto its range.

Proof. $A$ is continuous, which shows $\partial\left(B^{n}\left(Z^{m}, A\right)\right)$ closed; since $A$ is polonais, so are the groups involved, and the open mapping theorem applies.

Corollary. If $0 \rightarrow Z^{m} \rightarrow \boldsymbol{G} \rightarrow \boldsymbol{G} / \boldsymbol{Z}^{m} \rightarrow 0$ is exact and if the operation of $\boldsymbol{G}$ on the divisible, torsion-free, polonais $A$ is trivial when restricted to $Z^{m}$, there are spectral sequences $\mathbf{I}, \overline{\mathbf{I}}$, such that for all $p, q, \mathbf{I}_{2}^{p q}=H^{p}\left(\boldsymbol{G} / Z^{m}, H^{q}\left(Z^{m}, A\right)\right) \overline{\mathbf{I}}_{2}^{p q}=$ $\bar{H}^{p}\left(\boldsymbol{G} / \boldsymbol{Z}^{m}, \bar{H}^{q}\left(Z^{m}, A\right)\right)$ and if $Z^{m}$ is continuously embedded in $\boldsymbol{G}$, an isomorphism as above for the continuous groups.

Proof. Clear.

Definition. A vector space $V$ over a field $K$ is called linearly compact if $K$ has the discrete topology and if $V$ is a topological vector space over $K$, and further, for every set $\left(F_{\alpha}\right)_{\alpha \in A}$, of closed linear subvarieties of $V$, if $\cap F_{\alpha}$ is nonempty, $\alpha$ in a finite subset of $A$, then $\cap F_{\alpha_{\alpha \in A}}$ is nonempty. We also assume that $V$ is Hausdorff.

For a discussion of linearly compact vector spaces, see $[11$, p. 78]. The most. important properties of these spaces in which we are interested are listed below.

Fact. $V$ is linearly compact over $K$ iff $V \cong \prod_{\alpha} K_{\alpha}, K_{\alpha} \cong K$.

Fact. If $h:\left(V_{i}, f_{i j}\right) \rightarrow\left(W_{i}, g_{i j}\right)$ is a map of inverse systems, where each $V_{i}, W_{i}$ is a linearly compact vector space over $K$, then $\operatorname{Ker} h_{\infty} \cong \lim _{i} \operatorname{Ker} h_{i}$, and $\operatorname{Im} h_{\infty}$ $\cong \lim _{i} \operatorname{Im} h_{i}$.

For an indication of the proof, see [4, p. 226]. On the strength of these facts, we can prove the following useful

THEOREM. If $D$ is discrete and if $D \cong \lim ^{i} D_{i}$, and if $D$ operates trivially on the additive group of the discrete field $K$, then there is an isomorphism $H^{n}(D, K) \cong$ $\lim _{i} H^{n}\left(D_{i}, K\right)$.

Proof. We need only observe that $B^{n}(D, K)=\lim _{i} B^{n}\left(D_{i}, K\right)$ and that

$$
\text { ว: } B^{n}(D, K) \rightarrow B^{n+1}(D, K)
$$

is $\partial_{\infty}$ for the maps $\partial_{i}: B^{n}\left(D_{i}, K\right) \rightarrow B^{n+1}\left(D_{i}, K\right)$. This, together with the above facts, proves the theorem, if we note only that the $B^{n}\left(D_{i}, K\right), B^{n}(D, K)$ are all linearly compact.

COROLLARY. The same result holds true if $K$ is replaced by any linearly compact vector space over $K$.

THEOREM. If $D$ is discrete, $D \cong \lim ^{i} D_{i}$, and $A$ is compact, then there is an isomorphism $H^{n}(D, A) \cong \lim _{i} H^{n}\left(D_{i}, A\right)$.

Proof. Clear.

THEOREM. If $D \cong \lim ^{i} D_{i}$ is discrete and $V$ linearly compact over the discrete field $K, \bigwedge^{n}(D, V)=\lim _{i} \bigwedge^{n}\left(D_{i}, V\right)$ for all $n \geqq 1$. 
Proof. $Z^{n}(D, V)=\lim _{i} Z^{n}\left(D_{i}, V\right)$ and $\bigwedge^{n}(D, V)$ is just the image of the alternation map $A: Z^{n}(D, V) \rightarrow Z^{n}(D, V)$, but $A=A_{\infty}$, the limit of the alternation maps $A_{i}: Z^{n}\left(D_{i}, V\right) \rightarrow Z^{n}\left(D_{i}, V\right)$, and since all groups are linearly compact vector spaces over $K, \operatorname{Im} A_{\infty} \cong \lim _{i} \operatorname{Im} A_{i}$, which is just the statement of the theorem.

Definition ([15]). If $\boldsymbol{G}$ is totally disconnected and $p$ is a prime, $\boldsymbol{G}$ is called $p$ finite if there is a compact open subgroup $G_{0} \subset G$ and an integer $N$ such that for all open normal $G_{1} \subset G_{0}, p^{n}$ divides $\left[G_{0}: G_{1}\right]$ implies $n<N$.

THEOREM. If $V$ is a Fréchet space over a locally compact, non-archimedean polonais field $K$, and if $\boldsymbol{G}$ is a totally disconnected $p$-finite group, where $p$ is the characteristic of the residue class field of $K$, then $\bar{H}^{n}(G, V)=0$, for all $n$.

Proof. Monna and Springer in [15] have shown that in the above circumstances there is a Haar integral on $G$ for $K$-valued bounded Borel functions, and this proof extends naturally to functions which are bounded Borel and $V$-valued. If $f$ is in $\bar{Z}^{n}(G, V)$, define

$$
s f\left(g_{1}, \ldots, g_{n-1}\right)=\int_{G} g^{-1} f\left(g, g_{1}, \ldots, g_{n-1}\right) d g .
$$

Then $\partial(s f)=f$.

Using the ordinary Haar integral on a compact group $\boldsymbol{G}$ for $V$-valued bounded Borel functions, $V$ a Fréchet space over $R$, we get $\bar{H}^{n}(G, V)=0$ for all $n$.

LEMMA. If $V$ is a Fréchet space over $\boldsymbol{R}$ and $\boldsymbol{G} / \boldsymbol{H}$ is compact, we have an isomorphism $L^{n}(G, V)=L^{n}(H, V)$, where $L^{n}$ is the group of continuous functions on $G^{n}$ with values in $V$ which are homomorphisms in each variable separately, hereafter referred to as multilinear.

Proof. Since every $V$-valued character defined on a closed subgroup of $\boldsymbol{G}$ extends to $G, L^{1}(G, V)$ is exact in $G$, and the theorem is true for $n=1$. Assume the theorem true for $n$ and consider $L^{n+1}(G, V) \cong L^{1}\left(G, L^{n}(G, V)\right) \cong L^{1}\left(G, L^{n}(H, V)\right)$ $\cong L^{1}\left(H, L^{n}(H, V)\right)$ since $L^{n}(H, V)$ is Fréchet; this final group is just $L^{n+1}(H, V)$. This same method of proof can be used to show that for every abelian $A$, we have

$$
L^{n}(\boldsymbol{G}, \boldsymbol{A}) \cong L^{n}(\boldsymbol{G} /[\overline{\boldsymbol{G}, \boldsymbol{G}}], \boldsymbol{A}),[\boldsymbol{G}, \boldsymbol{G}]
$$

the commutator subgroup of $\boldsymbol{G}$.

COROLlaRY. Under the above assumptions, $\bigwedge^{n}(G, V) \cong \bigwedge^{n}(H, V)$.

COROLLARY. If $\boldsymbol{H}$ is compact instead of $\boldsymbol{G} / \boldsymbol{H}$, there are isomorphisms $L^{n}(\boldsymbol{G}, V)$ $\cong L^{n}(G / H, V), \bigwedge^{n}(G, V) \cong \bigwedge^{n}(G / H, V)$.

THEOREM. If $\boldsymbol{G}$ is a compactly generated abelian locally compact group and if $V$ is Fréchet over $R, \bar{H}^{n}(G, V) \cong \bigwedge^{n}(G, V)$.

Proof. There is an exact sequence $0 \rightarrow Z^{m} \rightarrow G \rightarrow K \rightarrow 0$, with $K$ compact. See $[6$, p. 86]. From a previous lemma, we get a spectral sequence for this extension 
and $\overline{\mathrm{I}}_{2}^{p q} \cong \bar{H}^{p}\left(K, \bar{H}^{q}\left(Z^{m}, V\right)\right), p+q=n$, say. Now since $\bar{H}^{q}\left(Z^{m}, V\right)$ is a Fréchet space over $R$, this is zero unless $p=0$, in which case $\bar{I}_{2}^{0 n} \cong \overline{\mathrm{I}}_{\infty}^{0 n} \cong \bar{H}^{n}(G, V) \cong \bar{H}^{n}\left(Z^{m}, V\right)$ $\cong \bigwedge^{n}\left(Z^{m}, V\right) \cong \bigwedge^{n}(G, V)$ by the previous lemma.

COROLlARY. If $\boldsymbol{G}$ is compactly generated as above, $V$ Fréchet over $\boldsymbol{R}$, then $\partial\left(\overline{\boldsymbol{B}}^{n}(\boldsymbol{G}, V)\right)$ is closed in $\overline{\boldsymbol{B}}^{n+1}(\boldsymbol{G}, V)$ and $\partial$ is open onto its image.

Proof. There is an exact sequence $0 \rightarrow \partial\left(\bar{B}^{n}(G, V)\right) \stackrel{i}{\rightarrow} \bar{Z}^{n+1}(G, V) \stackrel{A}{\rightarrow} \bigwedge^{n+1}(G V$, $\rightarrow 0$, and as everything is Fréchet, the open mapping theorem holds.

LEMMA. If $D$ is a discrete torsion group and $V$ is linearly compact over $K$, then $H^{n}(D, V)=0$, for all $n \geqq 1$.

Proof. $D \cong \lim ^{i} D_{i}, D_{i}$ finite. Then $H^{n}(D, V) \cong \lim _{i} H^{n}\left(D_{i}, V\right)=0$.

COROLlaRY. Let $\boldsymbol{G}$ be a union of compact open subgroups. Then there is an isomorphism $H^{n}(G, R) \cong 0$, for all $n \geqq 1$.

Proof. There is an exact sequence $0 \rightarrow H \rightarrow G \rightarrow G / H \rightarrow 0, H$ compact and open, $\boldsymbol{G} / \boldsymbol{H}$ discrete and torsion. We have a spectral sequence for this extension, and $\bar{I}_{2}^{p q} \cong \bar{H}^{p}\left(\boldsymbol{G} / \boldsymbol{H}, \bar{H}^{q}(\boldsymbol{H}, \boldsymbol{R})\right)$. This is zero for $q>0$, and for $q=0, \bar{H}^{p}(\boldsymbol{G} / \boldsymbol{H}, \boldsymbol{R})=0$, since $\boldsymbol{R}$ and $\bar{H}^{q}(\boldsymbol{H}, \boldsymbol{R})$ are linearly compact with the discrete topology on $\boldsymbol{R}$, the latter zero except when $q=0$.

THEOREM. Let $\boldsymbol{G}$ be locally compact abelian and let $\boldsymbol{G}_{\boldsymbol{K}}$ be the union of all compact subgroups of $\boldsymbol{G}$, which is just the set of compact elements of $\boldsymbol{G} . \boldsymbol{G}_{K}$ is a closed subgroup of $\boldsymbol{G}$ and

$$
\bar{H}^{n}(\boldsymbol{G}, \boldsymbol{R}) \cong \bar{H}^{n}\left(\boldsymbol{G} / \boldsymbol{G}_{K}, \boldsymbol{R}\right) .
$$

Proof. Since $H^{n}\left(\boldsymbol{G}_{K}, \boldsymbol{R}\right)=0$ and everybody is Fréchet, there is a spectral sequence for $0 \rightarrow \boldsymbol{G}_{K} \rightarrow \boldsymbol{G} \rightarrow \boldsymbol{G} / \boldsymbol{G}_{K} \rightarrow \mathbf{0}$ whose second term is $H^{p}\left(\boldsymbol{G} / \boldsymbol{G}_{K}, H^{q}\left(\boldsymbol{G}_{K}, \boldsymbol{R}\right)\right)$, which is zero unless $q=0$. Done.

THEOREM. If $\boldsymbol{G}$ is locally compact abelian, $\bar{H}^{n}(\boldsymbol{G}, \boldsymbol{R}) \cong \bigwedge^{n}(\boldsymbol{G}, \boldsymbol{R})$.

Proof. $G / G_{K} \cong R^{m} \times D$, where $D$ is discrete and torsion-free. But we can make a further reduction-there is an exact sequence

$$
0 \rightarrow Z^{m} \times D \rightarrow R^{m} \times D \rightarrow T^{m} \rightarrow 0 .
$$

Now since $Z^{m} \times D \cong \lim ^{i} Z^{n_{i}}$ and since $\bar{H}^{n}\left(Z^{m} \times D, R\right) \cong \lim _{i} \bar{H}^{n}\left(Z^{n_{i}}, R\right) \cong$ $\lim _{i} \bigwedge^{n}\left(Z^{n} i, R\right) \cong \bigwedge^{n}\left(Z^{m} \times D, R\right)$, we see that $\partial\left(\bar{B}^{n}\left(Z^{m} \times D, R\right)\right)$ is closed in $\bar{B}^{n+1}\left(Z^{m} \times D, R\right)$ and that $\partial$ is open onto its range. Thus there is a spectral sequence for the above extension. But because $T^{m}$ is compact, there are isomorphisms $\bar{H}^{n}(\boldsymbol{G}, \boldsymbol{R}) \cong \bar{H}^{n}\left(Z^{m} \times D, \boldsymbol{R}\right) \cong \bigwedge^{n}\left(Z^{m} \times D, R\right) \cong \bigwedge^{n}(\boldsymbol{G}, \boldsymbol{R})$.

THEOREM. If $\mathbf{0} \rightarrow \boldsymbol{H} \rightarrow \boldsymbol{G} \rightarrow \boldsymbol{G} / \boldsymbol{H} \rightarrow \mathbf{0}$ is exact, $\boldsymbol{H}$ abelian, and the restriction of the action of $\boldsymbol{G}$ on $\boldsymbol{R}$ to $\boldsymbol{H}$ is trivial, then there is a spectral sequence for this extension whose second term is $\bar{H}^{p}\left(G / H, \bar{H}^{q}(\boldsymbol{H}, \boldsymbol{R})\right)$. 
Proof. The fact $\bar{H}^{q}(\boldsymbol{H}, \boldsymbol{R}) \cong \bigwedge^{q}(\boldsymbol{H}, \boldsymbol{R})$ implies that $\partial$ is open and is onto its closed range $\partial\left(\bar{B}^{q-1}(G, R)\right)$.

Now if we consider $\prod_{i} \boldsymbol{R}_{i}$, each $\boldsymbol{R}_{i} \cong \boldsymbol{R}$, and the fact that each closed subspace of this product is also of this form, we also have that quotients are of this form, since everything splits. This allows us to see that $\bigwedge^{n}(D, \boldsymbol{R}) \cong \bar{H}^{n}(D, \boldsymbol{R}) \cong \prod_{i} \boldsymbol{R}_{i}$, for $D$ discrete torsion-free abelian. Now since $\prod_{i} \boldsymbol{R}_{i}$ is both a Fréchet space and a natural linearly compact space with the discrete topology on $R$, we see that all the proofs of the previous lemmas and theorems go through for $\prod_{i} \boldsymbol{R}_{i}$ instead of $\boldsymbol{R}$. From now on, we write simply $\prod \boldsymbol{R}$ for $\prod_{i} \boldsymbol{R}_{i}$.

Definition. $G$ is said to be continuously solvable of finite length if there is a sequence $\{e\} \subset G_{1} \subset \ldots \subset G$ of closed normal subgroups, such that $G_{i+1} / G_{i}$ is abelian and $\boldsymbol{G}_{i}$ is embedded continuously in $\boldsymbol{G}_{i+1}$.

THEOREM. If $G$ is continuously solvable of finite length, the natural homomorphism $H_{c}^{n}(\boldsymbol{G}, \Pi \boldsymbol{R}) \rightarrow \bar{H}^{n}(\boldsymbol{G}, \Pi \boldsymbol{R})$ is really an isomorphism, where $\boldsymbol{G}$ acts trivally on $\Pi \boldsymbol{R}$.

Proof. We use induction on the length of $G$, the case $n=1$ being that of $G$ abelian, already proved. Now from the previous theorem, there is a spectral sequence for the extension $0 \rightarrow G_{1} \rightarrow G \rightarrow G / G_{1} \rightarrow 0$, whose second term is $\bar{H}^{p}\left(G / G_{1}, \bar{H}^{q}\left(G_{1}, \Pi R\right)\right)$, for $p+q=n$, say. The transitivity of continuous embeddings tells us that $G_{1}$ is continuously embedded in $G$, and thus there is a spectral sequence for this extension for the continuous groups, as well. The natural homomorphism in the statement of the theorem induces maps for all $p, q:\left(\mathrm{I}_{\infty}^{p q}\right)_{c} \rightarrow \overline{\mathrm{I}}_{\infty}^{p q}$. If we can show that these are all isomorphisms for $\infty$ replaced by 2 , we are finished. But these maps are just

$$
H_{c}^{p}\left(G / G_{1}, H_{c}^{q}\left(G_{1}, \Pi R\right)\right) \rightarrow \bar{H}^{p}\left(G / G_{1}, \bar{H}^{q}\left(G_{1}, \Pi R\right)\right),
$$

or, since $H_{c}^{q}\left(\boldsymbol{G}_{1}, \Pi \boldsymbol{R}\right) \cong \bar{H}^{q}\left(\boldsymbol{G}_{1}, \Pi \boldsymbol{R}\right) \cong \prod \boldsymbol{R}$, and also

$$
H_{c}^{p}\left(\boldsymbol{G} / \boldsymbol{G}_{1}, \Pi \boldsymbol{R}\right) \rightarrow \bar{H}^{p}\left(\boldsymbol{G} / \boldsymbol{G}_{1}, \Pi \boldsymbol{R}\right)
$$

which are isomorphisms by the induction assumption, the length of $G / G_{1}$ being less than that of $\boldsymbol{G}$.

COROLlaRY. If $\boldsymbol{G}$ is a solvable Lie group, with a finite number of components, $H_{c}^{n}(\boldsymbol{G}, \boldsymbol{R}) \cong \bar{H}^{n}(\boldsymbol{G}, \boldsymbol{R})$.

Proof. $G$ is well known to be.continuously solvable.

Now let us investigate the groups $\bar{H}^{n}(G, A)$, where $G$ is a locally compact abelian group such that both $G$ and $\hat{G}$ are totally disconnected, and $A \cong Q_{p}$, the $p$-adic numbers.

THEOREM. If $\boldsymbol{G}$ is locally compact abelian as above, then there is an isomorphism $\bar{H}^{n}\left(G, Q_{p}\right) \cong H^{n}\left(G_{p}, Q_{p}\right)$, where $G_{p}$ is the p-primary component of $G$. 
Proof. From [2, p. 49] we can write $\boldsymbol{G}$ as $\boldsymbol{G}_{p} \times \Pi_{q \neq p} \boldsymbol{G}_{q}$, the latter group being the local direct product of the $q$-primary components $G_{q}$ with respect to certain compact open subgroups, $\boldsymbol{G}_{q}^{\prime}$. First we observe that $\bar{H}^{n}\left(\Pi_{q \neq p} \boldsymbol{G}_{q}, \boldsymbol{Q}_{p}\right)$ is zero since $\Pi_{q \neq p} G_{q}^{\prime}$ is $p$-finite and compact and since the discrete torsion group $\Pi_{q \neq p} \boldsymbol{G}_{q}$ modulo $\square_{q \neq p} G_{q}^{\prime}$ is the direct limit of finite groups, which are, of course, $p$-finite. Thus we have a spectral sequence for the subgroup $\square_{q \neq p} G_{q}$, and using this, we obtain the theorem.

Next, we let $A \cong T$ and we study $H^{2}(G, T)$.

Lemma. For arbitrary polonais $A, H_{c}^{2}(G, A) \rightarrow H^{2}(G, A)$ is one to one.

Proof. Let $f(x, y)=h(x)+h(y)-h(x y), f$ in $Z_{c}^{2}(G, A), h$ in $B^{1}(G, A)$. Now $h$ is continuous except on a set $P \subset G$ of first category. Let $y$ be not in $\bigcup_{n \geqq 0} x_{n}^{-1} P$, where $x_{n}$ is an arbitrary sequence in $\boldsymbol{G}$ approaching $x_{0}$. Then $h\left(x_{n}\right)=f\left(x_{n}, y\right)$ $-x_{n} h(y)+h\left(x_{n} y\right)$ which approaches $f\left(x_{0}, y\right)-x_{0} h(y)+h\left(x_{0} y\right)$ which is just $h\left(x_{0}\right)$, and $h$ is continuous.

LEMMA. If $G$ is abelian, $H^{2}(G, T)$ is Hausdorff.

Proof. A cocycle in $Z^{2}(G, T)$ is in $\partial\left(B^{1}(G, T)\right)$ iff it is symmetric, since $T$ is an injective for abelian groups.

LEMMA. If $\boldsymbol{G}$ is compact abelian, $\partial: \boldsymbol{B}^{1}(G, T) \rightarrow \partial\left(\boldsymbol{B}^{1}(G, T)\right)$ is open.

Proof. In this case, $B^{n}(G, T)$ is locally isomorphic to the Fréchet space $B^{n}(G, R)$, and the open mapping theorem applies.

COROLlaRY. If $0 \rightarrow \boldsymbol{H} \rightarrow \boldsymbol{G} \rightarrow \boldsymbol{G} / \boldsymbol{H} \rightarrow \mathbf{0}$ is exact with $\boldsymbol{H}$ compact abelian and the restriction of the operation of $\boldsymbol{G}$ on $T$ to $\boldsymbol{H}$ is trivial, there is a spectral sequence whose second term is $H^{p}\left(G / H, H^{2}(H, T)\right) \cong I_{2}^{p 2}$, for all $p \geqq 0$.

Proof. Clear.

THEOREM. If $\boldsymbol{G}$ is continuously solvable of finite length and acts trivially on $T$, then the natural homomorphism $H_{c}^{2}(G, T) \rightarrow H^{2}(G, T)$ is an isomorphism onto.

Proof. $H^{2}(G, T)$ can be identified with the set of equivalence classes of central extensions of $T$ by $G$ and $H_{c}^{2}(G, T)$ with those which split continuously, i.e., those central extensions

$$
0 \rightarrow T \rightarrow E \rightarrow G \rightarrow 0
$$

in which $T$ is continuously embedded. Then since we have already shown that the above homomorphism is one to one, the transitivity of continuous embeddings and the isomorphisms $H_{c}^{2}\left(R^{n}, T\right) \cong H^{2}\left(R^{n}, T\right), H_{c}^{2}(K, T) \cong H^{2}(K, T)$, for $K$ compact or discrete, show the theorem valid. These isomorphisms may be found in Kleppner, [9, Theorem 7.1, p. 33]. 
The only present thorn in the theory is $H^{3}(T, T)$, and by straightforward calculations, it is easy to show that there is a string of isomorphisms $H^{3}(\boldsymbol{R}, T) \cong$ $H^{3}(T, T) \cong \bar{H}^{4}(T, Z) \cong \bar{H}^{4}(R, Z)$. These groups are undoubtably all zero, and this fact would hasten the analysis of the groups $\bar{H}^{n}(G, Z)$ and $H^{n}(G, T), G$ abelian, at least. In a future paper, we shall obtain a spectral sequence for certain closed not necessarily normal subgroups of $\boldsymbol{G}$ along lines similar to the earlier sections here, and use it on simple groups.

ACKNOWLEDGEMENTS. I should like to express deep appreciation to my thesis adviser, Dr. Adam Kleppner, for the many hours he spent with me in discussing the problems that arose during the research for this thesis, in particular, for his important and interesting ideas concerning the notions of mathematical importance and interest.

Without the use of Dr. Calvin Moore's thesis, which he kindly lent me for a period of two years, and without the inspiring conversation about these matters of cohomology, much would be lacking in my research.

Also, I should like to thank Drs. Goldhaber, Horvath, Maltese, Mountjoy, Pukansky, Srinivasacharyulu, and the many others for their good will toward and interest in me while a graduate student at the University of Maryland.

\section{BIBLIOGRAPHY}

1. N. Bourbaki, Algèbre multilinéaire, Hermann, Paris, 1958.

2. J. Braconnier, Sur les groupes topologiques localement compacts, J. Math. Pures Appl. 27 (1948), 1-85.

3. S. Eilenberg and S. MacLane, Cohomology theory in abstract groups. I, Ann. of Math. (2) 48 (1947), 51-78.

4. S. Eilenberg, and N. E. Steenrod, Foundations of algebraic topology, Princeton Univ. Press, Princeton, N. J., 1952.

5. R. Godement, Topologie algébrique et théorie des faisceaux, Hermann, Paris, 1958.

6. E. Hewitt and K. A. Ross, Abstract harmonic analysis. I, Academic Press, New York, 1963.

7. G. Hochschild and J.-P. Serre, Cohomology of group extensions, Trans. Amer. Math. Soc. 74 (1953), 110-134.

8. J. L. Kelley and I. Namioka, Linear topological spaces, Van Nostrand, Princeton, N. J., 1963.

9. A. Kleppner, Multipliers on abelian groups, Math. Ann. 158 (1965), 11-34.

10. C. Kuratowski, Topologie, Vol. I, 4th ed., PWN, Warsaw, 1958.

11. S. Lefshetz, Algebraic topology,.Amer. Math Soc. Colloq. Publ., Vol. 27, Amer. Math. Soc., Providence, R. I., 1942.

12. G. W. Mackey, Induced representations of locally compact groups. I, Ann. of Math. (2) 55 (1952), 101-139.

13. - Les ensembles boréliens et les extensions des groupes, J. Math. Pures Appl. 36(1957), 171-178.

14. S. MacLane, Homology, Academic Press, New York, 1963.

15. A. F. Monna and T. A. Springer, Intégration non-archimédienne. I, Nederl. Akad. Wetensch. Proc. Ser. A 66=Indag. Math. 25 (1963), 634-642. 
16. C. C. Moore, Extensions and low dimensional cohomology theory of locally compact groups. I, II, Trans. Amer. Math. Soc. 113 (1964), 40-63, 64-86.

17. G. D. Mostow, Cohomology of topological groups and solvmanifolds, Ann. of Math. (2) 73 (1961), 20-49.

18. E. Snapper, Cohomology of permutation representations. I. Spectral sequences, J. Math. Mech. 13 (1964), 133-161.

\section{UNIVERSITY OF MARYLAND,}

Collage Park, Maryland 